\title{
Nonlinear conversion dynamics from self-trapped exciton states to a macroscopic photoinduced phase in strongly correlated organic radical crystals
}

\author{
Ikufumi Katayama, ${ }^{1}$ Toyoki Kon, ${ }^{2}$ Kei Mitarai, ${ }^{2}$ and Jun Takeda ${ }^{2}$ \\ ${ }^{1}$ Interdisciplinary Research Center, Yokohama National University, 79-5 Tokiwadai, Hodogaya-ku, Yokohama 240-8501, Japan \\ ${ }^{2}$ Department of Physics, Graduate School of Engineering, Yokohama National University, 79-5 Tokiwadai, \\ Hodagaya-ku, Yokohama 240-8501, Japan
}

(Received 15 June 2009; revised manuscript received 22 August 2009; published 17 September 2009)

\begin{abstract}
Nonlinear conversion from self-trapped exciton (STE) states with local lattice distortions to a macroscopic photoinduced phase has been investigated in strongly correlated organic radical 1,3,5-trithia-2,4,6triazapentalenyl crystals through luminescence and Raman-scattering measurements. The two-photon luminescence from STEs in the diamagnetic phase shows a quenching and a redshift with a clear threshold excitation photon density. The redshift directly represents a change in the local potential surface for the excited states, which stabilizes the excited domain and subsequently accelerates the macroscopic phase transition. The results strongly demonstrate that the local lattice distortions due to the STEs are the trigger for the photoinduced phase transition.
\end{abstract}

DOI: 10.1103/PhysRevB.80.092103

PACS number(s): 64.70.kt, 71.35.-y, 75.50.Xx, 78.55.Kz

Photoreactive materials showing photoinduced phase transition (PIPT) have been attracting great interest both for fundamental physics and various applications. ${ }^{1,2}$ The dynamics of the PIPT is especially fascinating because it involves nonlinear and nonequilibrium processes resulting from the cooperative interaction between the excited states. According to scenario of the PIPT dynamics predicted theoretically, the cooperative interaction induces an excited domain accompanied with local lattice distortions as a precursor of the PIPT. ${ }^{1,2}$ Above a threshold excitation photon density, the domain fully proliferates like a domino effect, leading to the macroscopic phase transition.

Although various materials such as spin-crossover complexes, ${ }^{3-5}$ organic ionic crystals,,${ }^{6,7}$ and organic polymer crystals $^{8}$ are found to show the PIPT, and extensive studies of these materials reveal the important features such as an existence of incubation time and a threshold excitation photon density to drive the PIPT, ${ }^{1,4}$ little is known about the excited domain, which is a precursor of the PIPT. This is partly because most of the PIPT materials have a small band gap which makes it difficult to study luminescence properties that reflect nature of the excited states with lattice distortions. Therefore, as far as we know, no direct observation has been reported so far that reveals an entire conversion dynamics from excited states with local lattice distortions to a macroscopic photoinduced phase via formation of the excited domain.

In this Brief Report, we will demonstrate that simple steady-state luminescence measurement can be a unique and powerful tool for understanding the entire and nonlinear PIPT behaviors because it can directly probe the excited states with the lattice distortions and evaluate a change in the local energy structure. By comparing the results on the luminescence measurements with those on the photoinduced Raman measurements, we could evaluate the PIPT dynamics.

Among the PIPT materials, we focus on organic radical 1,3,5-trithia-2,4,6-triazapentalenyl (TTTA) crystals that show the PIPT from diamagnetic low-temperature (LT) to paramagnetic high-temperature (HT) phase inside a hysteresis loop. ${ }^{9,10}$ TTTA has a magnetic bistability around room temperature ${ }^{11}$ and the paramagnetic HT phase is a Mott insulator due to a strong electron correlation between unpaired electrons in molecules. These unpaired electrons form dimers to make TTTA diamagnetic at low temperature. ${ }^{12}$ TTTA has a larger band gap $(\sim 1.7 \mathrm{eV})$ than the other PIPT materials (typically around $0.6 \mathrm{eV}$ ), which enables us to study the luminescence properties. Indeed, the luminescence from selftrapped excitons (STEs) was clearly observed in the LT phase with a large Stokes shift due to the strong electronlattice coupling. ${ }^{13,14}$

TTTA crystals were grown by the method previously reported. ${ }^{11,15}$ The crystals obtained are in the HT phase with typical size of $1.0 \times 0.3 \times 0.1 \mathrm{~mm}^{3}$. The LT phase crystals can be obtained by cooling the HT phase crystals to $77 \mathrm{~K}$ in liquid nitrogen. The LT phase crystals were excited by the laser light from an optical parametric oscillator pumped with an Nd:YAG laser. The output photon energy of the laser ranges from 0.56 to $1.38 \mathrm{eV}$ with a pulse duration of $5 \mathrm{~ns}$ and a repetition rate of $10 \mathrm{~Hz}$. After each one-pulse excitation on the LT phase crystal with the photon energy of $0.85 \mathrm{eV}$ and at a given photon density, we measured the luminescence spectrum with a single grating monochromator and a charge coupled device camera cooled at $223 \mathrm{~K}$. We use the same laser for the excitation of the luminescence but with sufficiently low power $\left(4.7 \times 10^{17}\right.$ photons $\left./ \mathrm{cm}^{2}\right)$ so that no PIPT occurs. Because of the strong scattered light from the fundamental laser pulses $(1.17 \mathrm{eV})$, the observed luminescence photon energy are limited above $1.3 \mathrm{eV}$. The wavelengths and sensitivities are corrected by $\mathrm{Hg}$ emission lines and a standard black body light source.

To estimate the conversion yield from the diamagnetic LT to the paramagnetic HT phase, we also measure the photoinduced Raman spectra of the sample by a Fourier-Raman spectrometer with a cw-Nd:YAG laser $(1.17 \mathrm{eV}) .{ }^{9}$ Because the excitation intensity of the cw-YAG laser is weak enough and the wavelength is much longer than the lowest absorption edge of both the HT and LT phases, we can measure the photoinduced Raman signals from a full volume of the 

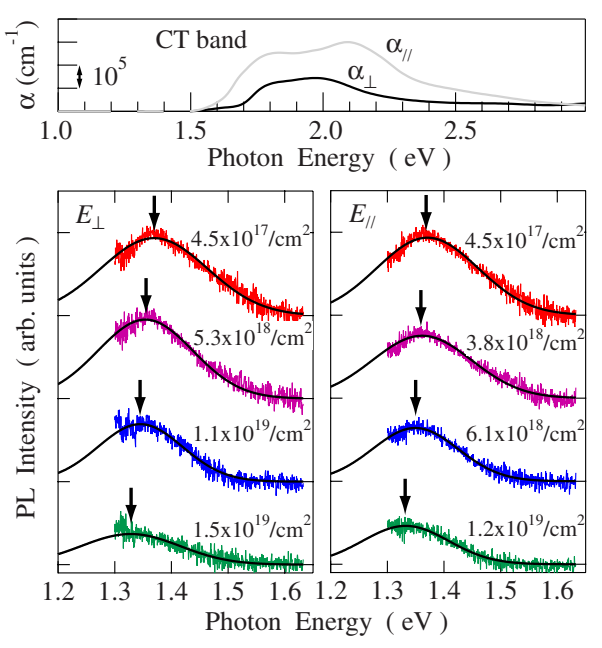

FIG. 1. (Color online) Two-photon luminescence spectra of the LT phase observed with polarizations perpendicular $\left(E_{\perp}\right)$ and parallel $\left(E_{\|}\right)$to the stacking axis after the single shot excitation with $0.85 \mathrm{eV}$ and given photon densities. Solid lines are the Gaussian fittings for the luminescence spectra after the reabsorption correction described in the text. The charge-transfer absorption bands of the LT phase are also shown on the top of the figure.

sample. All the experiments are performed at room temperature.

Figure 1 shows the photoluminescence (PL) spectra due to the STEs in the LT phase as a function of the excitation photon density. The absorption spectra of the LT phase are also shown on the top of the figure as a reference. Since it is difficult to estimate the two-photon absorption coefficients, we use the number of photons per unit area $\left(\right.$ photons $/ \mathrm{cm}^{2}$ ) as a unit of photon density, which is used in the references. ${ }^{6,13,16}$ The spectra are already corrected for reabsorption of the luminescence. Since the excitation energy is in the transparent region of the absorption spectrum of TTTA, a dominant excitation process is due to the two-photon absorption, leading to the homogeneous excitation as reported in the previous work. ${ }^{13}$ As shown in Fig. 1, the PL intensity and the peak energy decrease as the excitation photon density increases in both polarizations. Note that the observed luminescence is due to the LT phase but not due to the photoinduced HT phase which shows no luminescence in this energy range. Therefore, these experimental behaviors are related to the excited states of the LT phase.

In order to elucidate entire conversion mechanism from the LT to the HT phase, we compare the dependence on the excitation photon density of PL intensity with that of the conversion yield $\gamma$ to the HT phase, as shown in Fig. 2(a). Here, the PL intensity is normalized by the PL intensity before each one-pulse excitation on the LT phase crystal. On the other hand, the conversion yield $\gamma$ is estimated from the photoinduced Raman-scattering measurements by the same procedure previously reported. ${ }^{13}$ Since we measured the Raman spectra not only in the photoinduced phase but also both in the LT and HT phases, we could precisely evaluate the conversion yield from the LT to the HT phase. One can see that the PL intensity decreases with the excitation photon density having a threshold density of $3 \times 10^{18}$ photons $/ \mathrm{cm}^{2}$

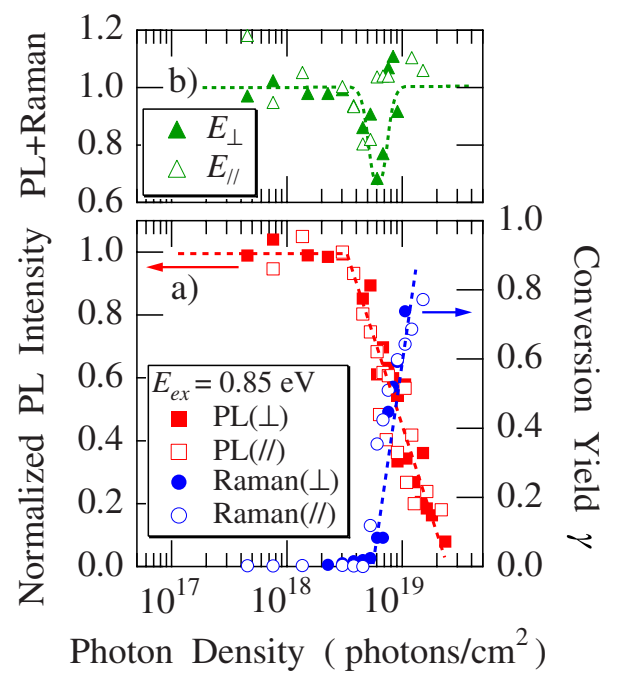

FIG. 2. (Color online) (a) Normalized PL intensity of the LT phase and the conversion yield $\gamma$ from the LT to the HT phase as a function of the excitation photon density. (b) Summation of the estimated conversion yield to the HT phase and the normalized PL intensity as a function of the excitation photon density. The dotted curves are visual guides for eyes.

while the conversion yield $\gamma$ increases having a slightly higher threshold photon density of $6 \times 10^{18}$ photons $/ \mathrm{cm}^{2}$, as shown by the dotted lines in Fig. 2(a).

In Fig. 2(b), we plot summation of the estimated conversion yield and the normalized PL intensity at given photon densities. The sum is almost unity below the threshold photon density of $3 \times 10^{18}$ photons $/ \mathrm{cm}^{2}$. With increasing the photon density, it once goes down to $\sim 0.7$ and then recovers to unity again as guided by the dotted curve in Fig. 2(b). This result is qualitatively explained as follows; when the LT phase is excited by a sufficiently weak photon density, the excited domain to drive the PIPT is not formed and the STE state is radiatively relaxed to the ground state of the LT phase. When the photon density becomes higher, the STEs become more stable because of lattice distortions due to the cooperative interaction between the excited states, resulting in the quenching and redshift of the STE luminescence. Since the excited domain does not sufficiently proliferate, the conversion to the macroscopic HT phase does not fully occur for these photon densities. Thus the sum might go down to $\sim 0.7$. With further increasing the photon density, proliferation of the excited states becomes to form an excited domain - that is, a precursor of the PIPT, leading to the effective conversion to a macroscopic HT phase. The value of the sum therefore returns back to unity. These results strongly show that the PL measurements coupled with the photoinduced Raman-scattering are powerful probes to evaluate not only the change in the potential surface for the excited states but also nonlinear conversion dynamics via formation of the excited domain.

To clearly show the change in the potential surface of the excited states in the LT phase which affects formation of the excited domain and the subsequent macroscopic phase transition, the peak energy of the STE luminescence as functions of the excitation photon density and the conversion yield $\gamma$ is 


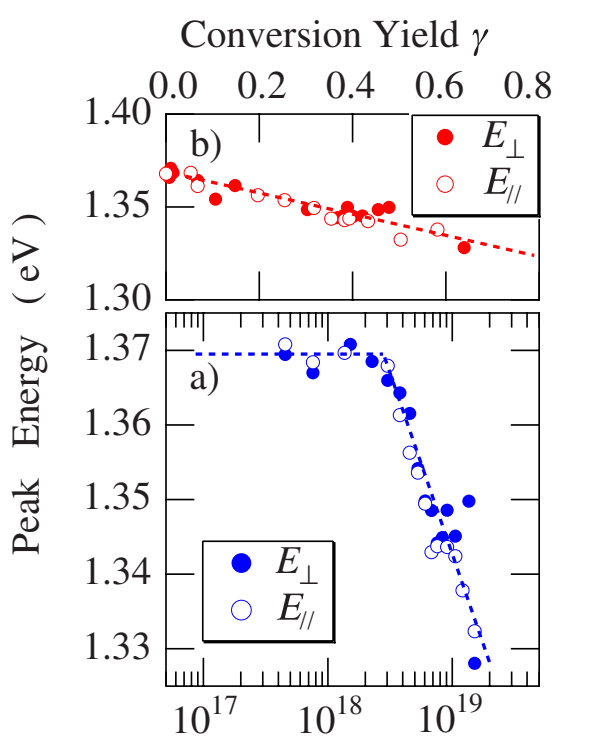

Photon Density ( photons $/ \mathrm{cm}^{2}$ )

FIG. 3. (Color online) (a) Peak energy of the STE luminescence as a function of the excitation photon density. (b) Peak energy shift of the luminescence during the PIPT as a function of the conversion yield. The broken line indicates a linear behavior of the energy shift.

depicted in Figs. 3(a) and 3(b). The peak energy shows a redshift as the photon density increases with a threshold photon density of $3 \times 10^{18}$ photons $/ \mathrm{cm}^{2}$ while it decreases in proportion to the conversion yield as shown by the dotted line. This linear behavior observed indicates that the change in the potential surface in the LT phase strongly depends on the fraction of the HT phase optically produced in the LT phase crystal.

According to the theory of domain formation in PIPT, ${ }^{17}$ the cooperative interaction between the excited states due to the coupling between lattice distortions stabilizes themselves by an amount of $-\Sigma_{l l^{\prime}} K_{l l^{\prime}} Q_{l} Q_{l^{\prime}}$, where $Q_{l}$ denotes a displacement of the interaction mode at each cites $l$ and $l^{\prime}$, and $K_{l l^{\prime}}$ the coupling constant. Here, we take account of this term to explain the redshift and quenching of the STE luminescence observed. Figures 4(a) and 4(b) show schematic energy diagrams of the STE luminescence without and with formation of the excited domains, respectively. The ground and excited states are assumed to have a similar potential curvature with a different local minimum for each energy surface. Here, we represent energy surfaces of the ground and excited states of the LT phase as $Q_{l}^{2}-2 Q_{\mathrm{g}} Q_{l}$ and $Q_{l}^{2}-2 Q_{\mathrm{ex}} Q_{l}+E_{\mathrm{FC}}\left(Q_{\mathrm{ex}}>Q_{\mathrm{g}}\right)$, respectively, when the excitation photon density is sufficiently weak.

With increasing the excitation photon density, the excited states become stabilized by the cooperative interaction. Assuming that the energy gain due to the cooperative interaction can be given by $-\gamma q Q_{l}$, where $\gamma$ denotes the fraction of the HT phase optically introduced into the LT phase crystal (conversion yield) and $q$ a constant of the proportionality, the local lattice distortion changes from $Q_{i}$ to $Q_{i}+(1 / 2) q \gamma$ $(i=\mathrm{g}, \mathrm{ex})$. Then the energy shift of the STE luminescence $\left(\Delta E_{\mathrm{STE}}=E_{0}^{\mathrm{Lumi}}-E^{\mathrm{Lumi}}\right.$ shown in Fig. 4$)$ is calculated to be (a)

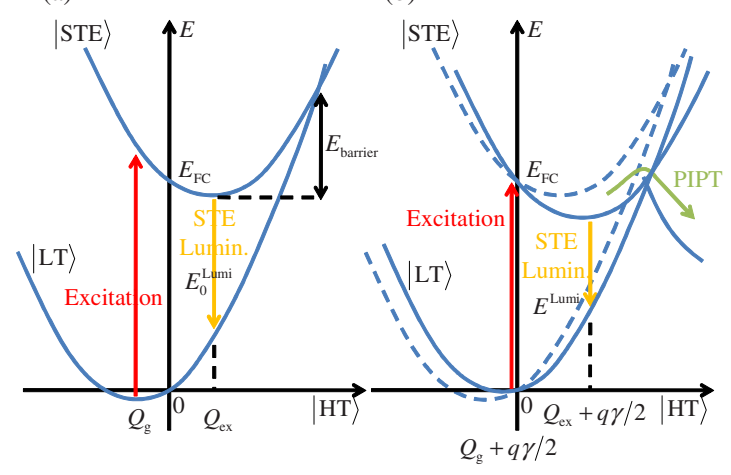

FIG. 4. (Color online) (a) Schematic energy diagram of the LT phase and the STE luminescence without cooperative interaction and (b) that with the cooperative interaction between the excited states on the basis of a model described in the text. Dotted lines are the same as the solid lines in (a). As the fraction of the HT phase increases, the excited states become stable, leading to the redshift and abrupt quenching of the STE luminescence. The relaxation pathway to the HT phase is also depicted. Notations of the variables are described in the text.

$-\left(Q_{\mathrm{ex}}-Q_{\mathrm{g}}\right) q \gamma$, that is, linear to the conversion yield $\gamma$. The redshift of the STE luminescence observed is therefore well explained by this simple model.

The change in the lattice distortions also leads to decrease in the barrier height $\left(E_{\text {barrier }}\right)$ for nonradiative relaxation pathways including the PIPT. According to the above simple model, the nonradiative decay rate from the excited to ground states exponentially increases with $\gamma$, which is given by $\exp \left[\frac{q \gamma}{k T}\left(\frac{E_{\mathrm{FC}}}{2\left(Q_{\mathrm{ex}}-Q_{\mathrm{g}}\right)}-Q_{\mathrm{ex}}\right)\right]$ under the first-order approximation of $q$. Similarly the nonradiative decay rate to the macroscopic HT phase might exponentially increase with $\gamma$. Therefore these nonradiative pathways must lead to the abrupt and sufficient decrease in the STE luminescence with increasing the excitation photon density.

The similar change in the local energy surface has been introduced by Hauser et al. ${ }^{18,19}$ to describe the relaxation dynamics in spin-crossover complexes; the decrease in the barrier height relevant to the density of the excited states induces the nonlinear and sigmoidal relaxation of photoexcited states. The similar mechanism might govern the nonlinear relaxation dynamics in TTTA, where the photoexcited STEs are affected by the local lattice distortions due to the phase conversion. Therefore, the STE states should be the trigger of the PIPT and the change in the potential surfaces due to cooperative interaction is the origin of nonlinearity in PIPT. Further elaborate theoretical model is highly desired to explain the entire nonlinear relaxation dynamics from local excited states to a macroscopic phase.

In summary, nonlinear conversion from the STE states with local lattice distortions to a macroscopic photoinduced phase has been demonstrated in strongly correlated TTTA crystals through steady-state luminescence and Ramanscattering measurements. We found that the STE luminescence in the LT phase shows an abrupt quenching and a 
redshift with a threshold excitation density while the fraction of the photoinduced HT phase increases with a slightly higher threshold density. These results suggest that the STE state interacting with local lattice distortions is the trigger for the PIPT. The observed redshift indicates the change in the local potential surface for the excited states in the LT phase, which stabilizes the excited domain to accelerate the macroscopic PIPT. The redshift and quenching properties of the STE luminescence can be qualitatively explained by a simple model. Because the luminescence measurements directly probe the change in the potential surface for the excited states with lattice distortions, they can be unique and power- ful spectroscopic tools for understanding the mechanism of PIPT.

This work was partly supported by the Special Coordination Funds for Promoting Science and Technology from Japan Science and Technology Agency (JST), the Grant-inAids for Scientific Research (A) from the Japan Society for the Promotion of Science (JSPS), and for Scientific Research on Innovative Areas "optical science of dynamically correlated electrons (DYCE)" (Grant No. 21104510) of the Ministry of Education, Culture, Sports, Science and Technology (MEXT).
${ }^{1}$ Photoinduced Phase Transitions, edited by K. Nasu (World Scientific, Singapore, 2004).

${ }^{2}$ K. Yonemitsu and K. Nasu, Phys. Rep. 465, 1 (2008).

${ }^{3}$ T. Tayagaki and K. Tanaka, Phys. Rev. Lett. 86, 2886 (2001).

${ }^{4}$ Y. Ogawa, S. Koshihara, K. Koshino, T. Ogawa, C. Urano, and H. Takagi, Phys. Rev. Lett. 84, 3181 (2000).

${ }^{5}$ S. Marcén, L. Lecren, L. Capes, H. A. Goodwin, and J. F. Létard, Chem. Phys. Lett. 358, 87 (2002).

${ }^{6}$ H. Okamoto, Y. Ishige, S. Tanaka, H. Kishida, S. Iwai, and Y. Tokura, Phys. Rev. B 70, 165202 (2004).

${ }^{7}$ O. Sato, T. Iyoda, A. Fujishima, and K. Hashimoto, Science 272, 704 (1996).

${ }^{8}$ S. Koshihara, Y. Tokura, K. Takeda, and T. Koda, Phys. Rev. Lett. 68, 1148 (1992).

${ }^{9}$ J. Takeda, M. Imae, O. Hanado, S. Kurita, M. Furuya, K. Ohno, and T. Kodaira, Chem. Phys. Lett. 378, 456 (2003).

${ }^{10}$ H. Matsuzaki, W. Fujita, K. Awaga, and H. Okamoto, Phys. Rev.
Lett. 91, 017403 (2003).

${ }^{11}$ W. Fujita and K. Awaga, Science 286, 261 (1999).

${ }^{12}$ K. Ohno, Y. Noguchi, T. Yokoi, S. Ishii, J. Takeda, and M. Furuya, ChemPhysChem 7, 1820 (2006).

${ }^{13}$ T. Kon, S. Oguri, I. Katayama, T. Kodaira, and J. Takeda, Phys. Rev. B 79, 035106 (2009).

${ }^{14}$ Y. Takahashi, T. Suemoto, S. Oguri, and J. Takeda, Phys. Rev. B 74, 193104 (2006).

${ }^{15}$ G. Wolmershäuser and R. Johann, Angew. Chem., Int. Ed. Engl. 28, 920 (1989).

${ }^{16}$ T. Suzuki, T. Sakamaki, K. Tanimura, S. Koshihara, and Y. Tokura, Phys. Rev. B 60, 6191 (1999).

${ }^{17}$ E. Hanamura and N. Nagaosa, J. Phys. Soc. Jpn. 56, 2080 (1987).

${ }^{18}$ A. Hauser, Chem. Phys. Lett. 192, 65 (1992).

${ }^{19}$ A. Hauser, J. Jeftić, H. Romstedt, R. Hinek, and H. Spiering, Coord. Chem. Rev. 190-192, 471 (1999). 